University of Wollongong

Research Online

Faculty of Engineering - Papers (Archive)

Faculty of Engineering and Information

Sciences

2001

\title{
Effect of uranium doping and thermal neutron irradiation on the flux-pinning of silver-clad Bi-Sr-Ca-Cu-O tapes
}

D. Marinaro

University of Wollongong

S X. Dou

University of Wollongong, shi@uow.edu.au

J. Horvat

University of Wollongong, jhorvat@uow.edu.au

J. Boldeman

ANSTO, Australia

R. Weinstein

University of Houston, USA

See next page for additional authors

Follow this and additional works at: https://ro.uow.edu.au/engpapers

Part of the Engineering Commons

https://ro.uow.edu.au/engpapers/21

\section{Recommended Citation}

Marinaro, D.; Dou, S X.; Horvat, J.; Boldeman, J.; Weinstein, R.; and Sawh, R.: Effect of uranium doping and thermal neutron irradiation on the flux-pinning of silver-clad Bi-Sr-Ca-Cu-O tapes 2001.

https://ro.uow.edu.au/engpapers/21

Research Online is the open access institutional repository for the University of Wollongong. For further information contact the UOW Library: research-pubs@uow.edu.au 
Authors

D. Marinaro, S X. Dou, J. Horvat, J. Boldeman, R. Weinstein, and R. Sawh 


\title{
Effect of Uranium Doping and Thermal Neutron Irradiation on the Flux-Pinning of Silver-Clad Bi-Sr-Ca-Cu-O Tapes
}

\author{
Damian G. Marinaro, Shi X. Dou, Josip Horvat, John Boldeman, Roy Weinstein and Ravi Sawh
}

\begin{abstract}
Ag/Bi-2223 tapes doped with small quantities of ${ }^{235} \mathrm{UO}_{4}$ powder were prepared by the powder-in-tube process and irradiated in a thermal neutron environment. Substantial improvements in critical current density (Jc) - applied field (H) performance and anisotropy have been previously reported. However, the radioactivity of the silver sheath is a limiting factor for commercial and industrial applications of this technique.

Here we report the performance of the technique using various doping levels (from 0.15 to 2 percent by weight (wt \%) $\left.\cup O_{4}\right)$ and thermal neutron fluences $\left(\Phi_{n}\right)$, in order to further reduce the silver radioactivity. Optimum fluence levels are identified and an optimum combination in terms of $\mathrm{Jc}$ - H performance is discussed. At a doping level of $2 \%{ }^{235} \mathrm{UO}_{4}$, a normalised $\mathrm{Jc}$ enhancement of 250 times is observed for an $0.8 \mathrm{~T}$ field aligned along the c-axis, and 25 times at $3 T$ along the abplane at $77 \mathrm{~K}$, compared to pre-irradiation values. At $0.6 \%$, these figures are 500 and 10 times, respectively.

The effects of the uranium doping and thermal neutron irradiation on the flux pinning strengths are also directly probed using dynamic relaxation techniques. The results show an increase in the effective pinning potential after doping and irradiation.
\end{abstract}

Index Terms-High Temperature Superconductors, Flux Pinning, Uranium Doping, Neutron Irradiation.

\section{INTRODUCTION}

$\mathrm{T}$ HE application of HTS tapes to industrial and commercial products is restricted by the maximum current that may be conducted by the tape within a typical working environment. Working environments may involve magnetic fields of different strengths as well as varying orientations relative to the superconducting matrix of the tape. It is therefore important to develop HTS tapes that have a low $J_{c}$ degradation under an applied field and a low $\mathrm{J}_{\mathrm{c}}$ dependence on the field orientation.

Manuscript received August 10, 2000. This work was supported in part by the Australian Institute of Nuclear Science and Engineering and the Australian Research Council.

D. G. Marinaro, S. X. Dou, and J. Horvat are with the Institute for Superconducting and Electronic Materials, University of Wollongong, Wollongong, NSW 2500, Australia (telephone: 42215730, e-mail: dgm01@uow.edu.au.

J. Boldeman is with the Australian Nuclear Science and Technology Organisation, Lucas Heights, NSW, 2534, Australia (e-mail: jwb@ansto.gov.au).

R. Weinstein and R. Sawh are with the Institute of Beam Particles, University of Houston, Houston, TX 80309 USA (e-mail: weinstein@uh.edu).
The $\mathrm{J}_{\mathrm{c}}$ and $\mathrm{J}_{\mathrm{c}}-\mathrm{H}$ performance of HTS materials is limited by weak links at grain boundaries and by the motion of magnetic flux lines that penetrate the HTS material. Over the course of the last decade, much progress has been made into the weak link problem, improving the zero-field $J_{c}$ to a level approaching the requirements for some commercial applications. Improvements in grain connectivity, texture and phase composition are some of the areas where progress has been made [1], [2]. The problem of flux pinning, however, is not well resolved by these materials processing technologies for technical applications.

One method used to improve the pinning properties of HTS materials is the introduction of structural defects into the crystalline lattice [3]. The defects suppress the local superconducting order parameter. Magnetic flux lines are then pinned in the region of damage because the energy associated with magnetic flux penetration into superconductor is smaller in this region with weaker superconductivity.

Irradiation techniques can be used to create the structural defects. Irradiating a HTS sample with a fluence of heavy ions can create a columnar track of damage which acts as a strong pinning centre, but all the tracks are aligned along the direction of the incident ion beam and so pinning is effective only for specific field orientations [4]. Also, the tracks do not penetrate very far into the material because of the high stopping energy, so that pinning is limited to a surface layer. Fast neutron [5] or proton [6] irradiation is used to create damage centres within the entire sample volume, forming columnar defects. However they are not a cost-effective way of improving the flux pinning.

Ideally, defect centres should be uniformly located throughout the material, should have a columnar shape that maximises the pinning strength, and they should be oriented in random directions to reduce any vortex motion by kinksliding between parallel defects. By doping the superconductor precursor powder with a small amount of ${ }^{235} \mathrm{U}$ and then irradiating with a thermal-neutron fluence, $\Phi_{\mathrm{n}}$, after processing [7], the fragments resulting from fission of the uranium can produce randomly splayed, roughly columnar tracks throughout the superconductor matrix.

This method has already produced very promising results in terms of $\mathrm{J}_{\mathrm{c}}-\mathrm{H}$ performance in Y123 and other HTS [8], particularly $\mathrm{Ag} / \mathrm{Bi}-2223$ tapes [9]. The major shortcoming of this method is the induced radioactivity of the silver sheath. Silver has a moderate thermal-neutron capture cross-section, decaying after neutron capture via $\beta^{-}$and $\gamma$ emission. 
Minimising $\Phi_{n}$ will minimise the activity of the silver sheath, but it will also reduce the density of fission tracks, and hence the density of flux pinning centres. As the density of tracks is proportional to the total mass of ${ }^{235} \mathrm{U}$, times $\Phi_{\mathrm{n}}$, then a reduction in $\Phi_{\mathrm{n}}$ can be compensated by an increase in ${ }^{235} \mathrm{U}$. However, as will be shown, an increase in ${ }^{235} U$ leads to a degradation in the zero-field $J_{c}$. A compromise level must be thus ascertained where the level of radioactivity can be kept as low as possible (low $\Phi_{n}$ ) while still maintaining significant improvements in $\mathbf{J}_{\mathrm{c}}$ at zero field and $\mathbf{J}_{\mathrm{c}}-\mathrm{H}$ performance. The principle aim of this paper is to study the effects of reducing $\Phi_{\mathrm{n}}$ and increasing the percentage of ${ }^{235} \mathrm{U}$ on the flux pinning behaviour of $\mathrm{Ag} / \mathrm{Bi}-2223$ tapes, by using a wide range in ${ }^{235} \mathrm{UO}_{4}$ doping and neutron fluence.

The flux pinning strength can be probed more directly by dynamic magnetic-moment relaxation methods, as discussed in [10]. We begin with the relation [11]

$$
\frac{d\langle B\rangle}{d t}=\frac{2 H a v_{0}}{d} \exp \left[\frac{-U_{e f f}(j, H)}{k T}\right] \text {, }
$$

where $\langle B\rangle$ is the average flux density, $a$ is the flux hop distance, $v_{0}$ is an attempt frequency, $d$ is a characteristic sample thickness and $U_{\text {eff }}$ is an effective energy barrier to the motion of flux lines. Expanding the flux density in terms of the sample magnetisation, $M$, and the applied magnetic field, (1) can be rearranged to give

$$
U_{e f l}(j, H)=k T\left[\ln \left(\frac{H}{\dot{H}}\right)+\ln \left(\frac{2 a v_{\theta}}{d}\right)\right]
$$

Here, use is also made of the assumption that by sweeping the field over a single hysteresis loop, at a constant sweep rate, $4 \pi \mathrm{d} M / \mathrm{d} t \ll \mathrm{d} H / \mathrm{d} t$. If we plot the calculated $\mathrm{U}_{\text {eff }}$ against $\mathrm{M}-\mathrm{M}_{\mathrm{eq}}$ (giving the irreversible magnetisation of the sample, $M_{\text {irr) }}$ and assume that $M_{\text {irr }} \propto J$, we obtain the approximate dependence of the effective activation energy on the current density. By measuring hysteresis loops at various temperatures and sweep rates, we can probe $\mathrm{U}_{\text {eff }}$ over a range of $\mathrm{M}_{\text {irr. }}$.

The second logarithmic term in (2) is essentially constant at fixed field and sweep rate. By requiring the continuity of $\mathrm{U}_{\text {eff }}$, the value of the constant $C=\ln \left(2 a v_{0} / d\right)$, can be estimated by plotting $\ln H-\ln [\mathrm{d} H / \mathrm{d} t]$ against $\mathrm{M}_{\mathrm{irr}}$, for different $\mathrm{d} H / \mathrm{d} t$ and $T$.

\section{EXPERIMENTAL PROCEDURE}

\section{A. Samples}

Multi-filamentary Ag/Bi2223 tapes were fabricated using the P-I-T process [2]. Precursor powders were doped with a series of weight percentages of ${ }^{235} \mathrm{UO}_{4}(0.15,0.6$ and 2- $\mathrm{wt} \%)$ prior to processing.

\section{B. Irradiation}

Tapes were packed in sets of three where possible: two tapes of a particular doping percentage, together with a nondoped tape. Each set was irradiated by highly moderated thermal neutrons at the HIFAR reactor of the Australian Nuclear Science and Technology Organisation, to various $\Phi_{n}$. The combinations of ${ }^{235} \mathrm{UO}_{4}$ and $\Phi_{\mathrm{n}}$ are shown in Table I.

\section{Transport Measurements}

Transport measurements of $J_{c}-H$ and $J_{c}-\theta$ (where $\theta$ is the angle of field orientation relative to the c-axis) from 0 to 1 Tesla were conducted on a $1.2 \mathrm{~T}$ electromagnet using a fourprobe method and a $1 \mu \mathrm{V} / \mathrm{cm}$ criterion. High field measurements of $\mathrm{J}_{\mathrm{c}}-\mathrm{H}$ were performed on a $12 \mathrm{~T}$ superconducting magnet. All measurements were performed at $77 \mathrm{~K}$.

\section{Magnetic Measurements}

Magnetic hysteresis loops for the determination of $U_{\text {eff }}$ were performed in an Oxford Instruments VSM on two samples cut from tapes that had been doped to 0.6- wt \%. One tape had been irradiated to $\Phi_{\mathrm{n}}=2.25 \times 10^{19}$ neutrons $/ \mathrm{m}^{2}$, while the other remained non-irradiated. The measurements were taken at temperatures ranging between 77 and $15 \mathrm{~K}$, and at sweep rates of $0.05,0.1,0.25 .0 .5,0.75$ and $1.0 \mathrm{~T} / \mathrm{min}$.

\section{RESULTS AND DISCUSSION}

Fig. 1 shows the $J_{c}-H$ performance of the tapes, for field orientations parallel and perpendicular to the c-axis of symmetry of each tape (running perpendicular to the tape surface). From these plots, the effects of altering the doping percentages and neutron fluence levels can be clearly seen.

The first noticeable feature is that the zero-field critical current density, $\mathrm{J}_{\mathrm{c} 0}$, is reduced for the tapes with 2- wt $\%$ doping. The $\mathrm{J}_{\mathrm{c} 0}$ for each tape is shown in Table I. This reduction in $J_{c 0}$ is due to the inclusion of the uranium within the superconductor matrix. Efforts are under way to reduce this initial degradation by changing the methods by which the ${ }^{235} \mathrm{U}$ is added to the precursor powder [12]. The current method of adding ${ }^{235} \mathrm{UO}_{4}$ powder therefore limits the effectiveness of increasing doping and reducing irradiation.

The best performance of critical current in both parallel and perpendicular applied fields is for a doping of $0.6-\mathrm{wt} \%$ and $\Phi_{n}=3 \times 10^{19} \mathrm{~m}^{-2}$. Then $J_{c}$ is 25 times larger than for a non-irradiated tape at $5 \mathrm{~T}$ (perpendicular to the c-axis) and at $500 \mathrm{mT}$ ( parallel to c).

TABLE I Uranium Doping Percentages and Total IRRadiation Fluence

\begin{tabular}{ccc}
\hline $\begin{array}{c}\text { Doping Percentage } \\
(\text { wt } \%)\end{array}$ & $\begin{array}{c}\text { Thermal Neutron Fluence } \\
\left(10^{19} \mathrm{~m}^{-2}\right)\end{array}$ & $\begin{array}{c}\text { Zero Field } \mathrm{J}_{\mathrm{c} 0} \\
\left(10^{7} \mathrm{Am}^{-2}\right)\end{array}$ \\
\hline 0 & 0 & 24.4 \\
0 & 2.25 & 22.9 \\
0.15 & 0 & 23.6 \\
0.15 & 6 & 17.8 \\
0.6 & 0 & 22.4 \\
0.6 & 2.25 & 18.1 \\
0.6 & 3 & 16.6 \\
0.6 & 4 & 10.4 \\
2 & 0.05 & 13.0 \\
2 & 0.2 & 13.0 \\
2 & 1 & 10.0 \\
\hline
\end{tabular}




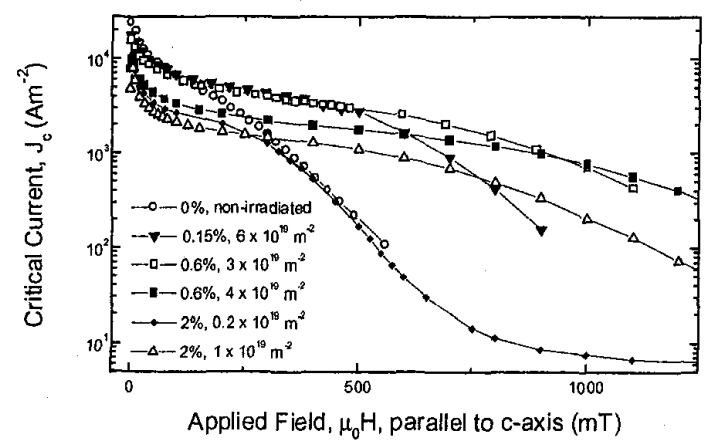

a) aligned perpendicular to $\mathrm{c}$-axis, along the ab-plane. The effects of Uranium doping and irradiation at different levels can be clearly seen

A slightly higher $\Phi_{\mathrm{n}}$ of $4 \times 10^{19} \mathrm{~m}^{-2}$ resulted in a reduced $\mathrm{J}_{\mathrm{c}}$, probably due to excessive damage to the superconductor matrix. In terms of absolute $J_{c}$ performance, $\Phi_{\mathrm{n}}=3 \times 10^{19} \mathrm{~m}^{-2}$ appears to be an optimum level before degradation occurs. However, the overall pinning improvement is greater for the higher $\Phi_{\mathrm{n}}$. This can be seen by plotting the normalised critical current density, $J_{\mathrm{c}} / J_{\mathrm{c} 0}$, as in Fig. 2 . These show the changes in $\mathrm{J}_{\mathrm{c}}$ caused by increasing the applied field. Improvements in pinning result in a weaker field dependence of $J_{c} / J_{c 0}$. The best performance, in terms of the weakest field dependence, is then for a 0.6- wt $\%$ doping and $\Phi_{n}=4 \times 10^{19} \mathrm{~m}^{-2}$ for either fields oriented along the c-axis, or fields greater than $7 \mathrm{~T}$ oriented perpendicular to the c-axis. However, $\mathrm{J}_{\mathrm{c} 0}$ decreases with the radiation damage. Because of this, the best performance in terms of $J_{c}$ for fields perpendicular to c-axis was obtained for $0.6 \%$ - wt and $\Phi_{\mathrm{n}}=3 \times 10^{19} \mathrm{~m}^{-2}$, as seen in Fig. 1.

The performance of tapes doped with 2- wt\% of $\mathrm{UO}_{4}$ is improved with increasing irradiation. After irradiation to 0.2 $\mathrm{x} 10^{19} \mathrm{~m}^{-2}$, the tape performance returns approximately to predoping levels, and after a $1 \times 10^{19} \mathrm{~m}^{-2}$ irradiation, the performance approaches that for a $6 \times 10^{19} \mathrm{~m}^{-2}$ irradiation on a tape doped with $0.15-\mathrm{wt} \%$. Thus for a suitable increase in ${ }^{235} \mathrm{U}$, the irradiation level can be substantially reduced while

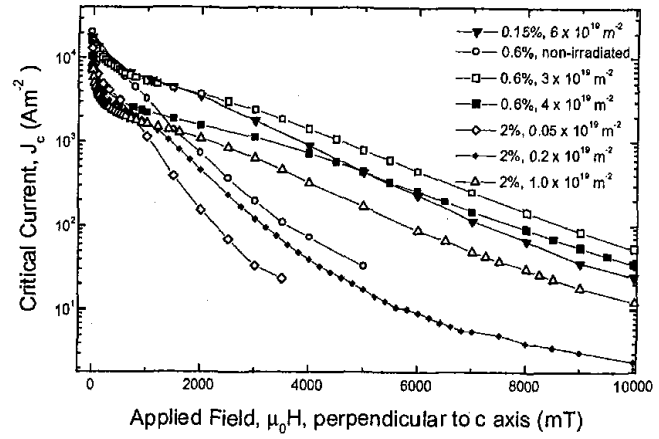

maintaining similar improvements in flux pinning. In this case, the irradiation fluence is 6 times lower, which results in a very significant drop in the radioactivity of the silver sheath.

The level that is considered as optimum for an industrial application depends entirely on any restrictions or requirements placed upon the tape by the particular application. If total available critical current over a wide range of applied fields is important, then a moderate uranium doping, followed by a moderate thermal neutron irradiation (for example, $0.6-w t \%$ and $3 \times 10^{19} \mathrm{~m}^{-2}$ ) provides significant improvements in $J_{c}-H$ performance. The level of silver radioactivity produced is still quite high though.

If, on the other hand, improvements in flux pinning are required and moderate $J_{c}$ degradation is acceptable, but minimisation of silver radioactivity is important, then a higher percentage of ${ }^{235} U$ and a low (but still sufficient) $\Phi_{n}$ is best. If changes to the processing procedure can reduce the degradation of $\mathrm{J}_{\mathrm{c} 0}$ caused by the addition of ${ }^{235} \mathrm{U}$ compounds, then this combination would be optimal for most applications. This is also the optimal case if simple reduction in the anisotropic behaviour of the tapes is required in low applied fields. Fig. 3 shows an example of the angle dependence of $J_{c}$

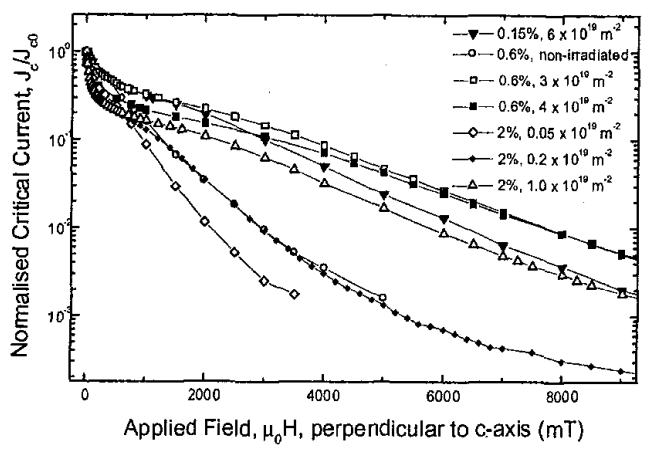

b)

a)

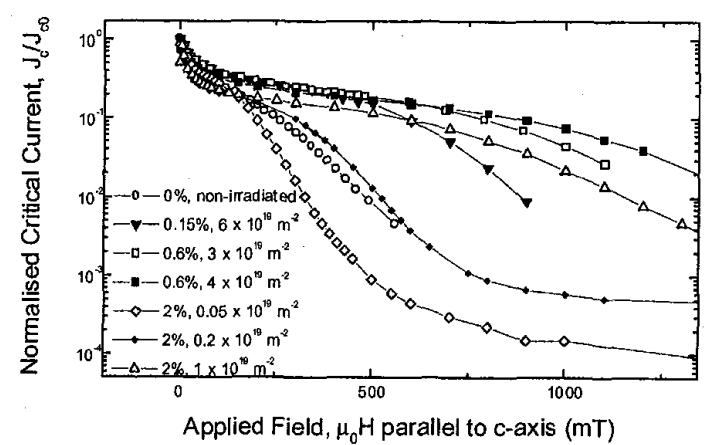

Fig. 2. Magnetic field dependence of $\mathbf{J}_{\mathfrak{c}}$ normalised to the zero field $\mathbf{J}_{\mathfrak{c} 0}$, for a) field parallel to c-axis, and b) field perpendicualr to c-axis. These figures highlight more accurately the effects of improvements in flux pinning. 


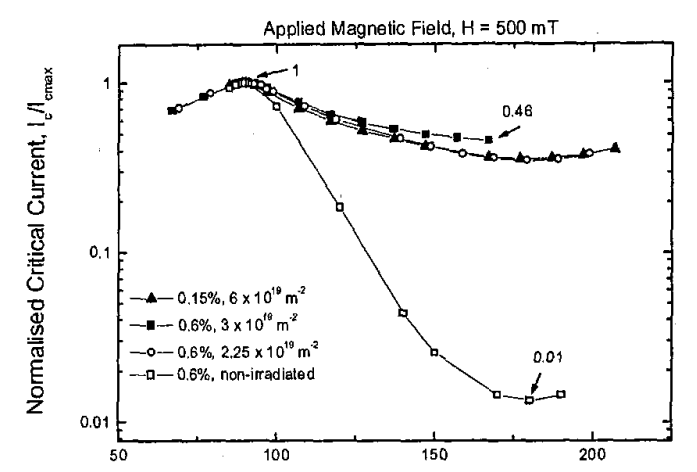

Angle of Applied Magnetic Field, relative to c-axis (degrees)

Fig. 3. Angular dependence of $\mathrm{J}_{\mathcal{C}}$, normalised to maximum current for field perpendicular to c-axis.

in a $500 \mathrm{mT}$ applied field, relative to the c-axis. Anisotropy, for a $0.6-$ wt $\%$ doping and $\Phi_{\mathrm{n}}=3 \times 10^{19} \mathrm{~m}^{-2}$ is reduced by 46 times.

The flux pinning strength, $\mathrm{U}_{\text {eff, }}$ was probed for two tapes doped with $0.6-\mathrm{wt} \%{ }^{235} \mathrm{UO}_{4}$, one before irradiation and the other after irradiation to $\Phi_{\mathrm{n}}=2.25 \times 10^{19} \mathrm{~m}^{-2}$, and at an applied field of $500 \mathrm{mT}$ parallel to the c-axis. The current dependence of $U_{\text {eff }}$ is shown in Fig. 4. The values of $C$ required for continuity were 18 and 25 for the non-irradiated and irradiated tapes, respectively. Temperature scaling was also required for continuity [13]. The activation energy was multiplied by $\mathrm{g}(t)=\left(1-t^{2}\right)^{-1}$, where $t=T / T_{c}$, to give good continuity at low temperatures.

We can see that there is a definite improvement in flux pinning energy after irradiation. At high currents the energy after irradiation is about one-and-a-half times as great, while at low currents $U_{\text {eff }}$ is twice that before irradiation. The constant $\mathrm{C}$ also changes with irradiation. This change in $\mathrm{C}$ represents a change in $a v_{0}$ with irradiation of approximately $\mathrm{e}^{7}(\sim 1100)$ times. Thus the combination of flux hopping distance and attempt frequency changes after ${ }^{235} \mathrm{U}$ doping and thermal neutron irradiation.

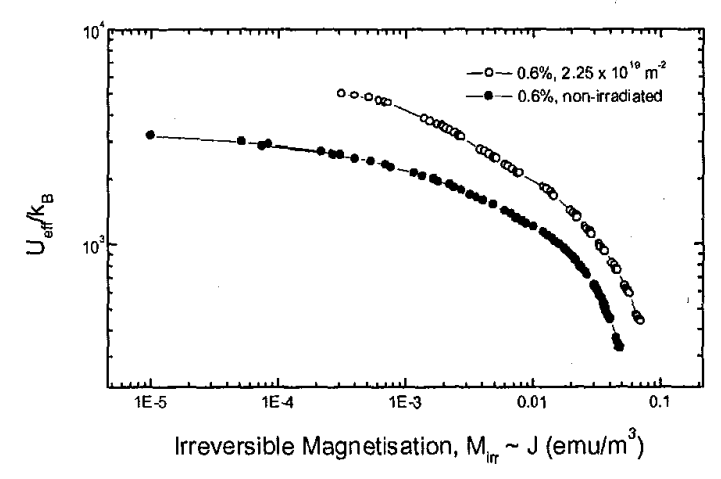

Fig. 4. Effective activation energy, $U_{\text {eff }}(\mathrm{J})$, of two ${ }^{235} U$ doped tapes, at an applied field of $500 \mathrm{mT}$ parallel to the c-axis. Each set of points represents the results from different sweeping rates at a single temperature. The lowest isotherm, $15 \mathrm{~K}$, is on the far right (highest $\mathrm{M}_{\mathrm{irr}}$ ).

\section{CONCLUSION}

The addition of ${ }^{235} \mathrm{UO}_{4}$ to $\mathrm{Bi}-\mathrm{Sr}-\mathrm{Ca}-\mathrm{Cu}-\mathrm{O}$ precursor powder, and irradiation with thermal neutrons after processing of the tape, improves the flux pinning properties of the superconductor. Zero field critical current is adversely affected by the ${ }^{235} \mathrm{UO}_{4}$ addition, so there is a limit to the amount by which a tape can be doped. Neutron-capture by the silver sheath and the subsequent $\gamma$ emission also limits the $\Phi_{n}$ to which a tape may be irradiated.

A compromise must be found, depending on the requirements for the intended application of the tape. If large improvements in $J_{c}-H$ are sought, moderate ${ }^{235} U$ and $\Phi_{n}$ levels provide excellent performance. Much lower radioactivity levels can be achieved with high ${ }^{235} \mathrm{U}$ content and low $\Phi_{\mathrm{n}}$ while still retaining the improved flux pinning performance, but $J_{c}$ levels are much lower as well. Anisotropy can also be greatly reduced by this doping method.

\section{REFERENCES}

[1] S. X. Dou, Q. Y. Hu, Y. C. Guo, J. Horvat and H. K. Liu, "Cryogenic deformation process of high temperature superconductors," Supercond. Sci. Technol. vol. 11, pp. 781-787, 1998.

[2] R. Zeng, B. Ye, J. Horvat, Y. C. Guo, B. Zeimetz, X. F. Yang, T. P. Beales, H. K. Liu and S. X. Dou, "Grain connectivity and flux pinning in Bi-2223/Ag tapes," Physica C, vol. 307, pp. 29-45, 1998.

[3] S. X. Dou, X. L. Wang, Y. C. Guo, Q. Y. Hu, P. Mikheenko, J. Horvat, $\mathrm{M}$. Ionescu and H. K. Liu, "Introduction of pinning centres into $\mathrm{Bi}-(\mathrm{Pb})-\mathrm{Sr}-\mathrm{Ca}-\mathrm{Cu}-\mathrm{O}$ superconductors," Supercond. Sci. Technol., vol. 10, pp. 1-16, 1997.

[4] L. Civale, A. D. Marwick, T. K. Worthington, M. A. Kirk, J. R. Thompson, L. Krusin-Elbaum, Y. Sun, J. R. Clem and F. Holtzberg, "Vortex confinement by columnar defects in YBaCuO crystals", Phys. Rev. Lett., vol. 67, pp. 648-651, 1991.

[5] A. Umezawa, G. W. Crabtree, J. Z. Liu, H. W. Weber, W. K. Kwok, L H. Nunez, T. J. Moran, C. H. Sowers and H. Claus, "Enhanced critical magnetization currents due to fast neutron irradiation in single-crystal YBaCuO", Phys. Rev. B, vol. 36, pp. 7151-7154, 1987.

[6] L. Civale, A. D. Marwick, M. W. McElfresh, T. K. Worthington, A. P. Malozemoff, F. H. Holtzberg and M. A. Kirk, "Defect independence of the irreversibility line in proton-irradiated Y-Ba-Cu-O crystals", Phys. Rev. Lett, vol, 65, pp. 1164-1167, 1991.

[7] R. Weinstein, Y. Ren, J. Liu, I. G. Chen, R. Sawh, C. Foster, and V. Obot, Advances in Superconductivity $V i$ (Proc. of $6^{\text {th }}$ Internat. Sympos. on Superconductivity, Hiroshima), p. 855, Springer-Verlag, 1993

[8] R. Weinstein, R. Sawh, Y. Ren, and D. Parks, Materials Science and Engineering, vol. B53, p. 38, 1988.

[9] G. W. Schulz, C. Klein, H. W. Weber, S. Moss, R. Zeng, S. X. Dou, R. Sawh, Y. Ren and R. Weinstein, "Enhancement of transport current densities in Bi-2223 tapes by fission tracks," Appl. Phys. Lett., vol. 73, p. $3935,1998$.

[10] D. Marinaro, S. X. Dou, J. Horvat, Y. C. Guo, J. Boldeman, A. Gandini, R. Sawh, Y. Ren and R. Weinstein, "The effects of uranium doping and thermal neutron irradiation on the pinning properties of $\mathrm{Ag} / \mathrm{Bi}-2223$ tapes," Physica C, submitted for publication.

[11] H. L. Ji, Z. X. Shi, Z. Y. Zeng, X. Jin, X. X. Yao, X. S. Rong, Y. M. Ni and Z. X. Zhao, "Equivalent approaches to studying flux creep" Physica C, vol. 217, pp. 127-134, 1993.

[12] D. Milliken, J. H. Ahn and S. X. Dou, "Phase formation and thermal treatment optimisation in uranium compound doped $\mathrm{Bi}_{2} \mathrm{Sr}_{2} \mathrm{Ca}_{2} \mathrm{Cu}_{3} \mathrm{O}_{\mathrm{x}} / \mathrm{Ag}$ superconducting tapes", Physica $C$, in press.

[13] M. E. McHenry, S. Simizu, H. Lessure, M. P. Maley, J. Y. Coulter, I. Tanaka and $\mathrm{H}$. Kojima, "Dependence of the flux-creep activation energy on the magnetisation current for a $\mathrm{La}-\mathrm{Sr}-\mathrm{Cu}-\mathrm{O}$ single crystal," Phys. Rev. B, vol.44, pp. 7614-7624, 1991. 